\title{
Publisher's Note: Effect of reaction-step-size noise on the switching dynamics of stochastic populations [Phys. Rev. E 93, 052117 (2016)]
}

Shay Be'er, Metar Heller-Algazi, and Michael Assaf

(Received 28 June 2016; published 5 July 2016)

DOI: 10.1103/PhysRevE.94.019901

This paper was published online on 9 May 2016 with errors in an inline equation and in Eqs. (2) and (3). On page 1, last line in the right-hand column, the first relation should read as $2 A \stackrel{2 \alpha / N}{\longrightarrow} 3 A$. Equation (2) should read as

$$
\dot{P}_{n}=\frac{k_{0}}{\langle k\rangle}\left[\sum_{k=0}^{n} D(k) P_{n-k}-\sum_{k=0}^{\infty} D(k) P_{n}\right]+\frac{\alpha(n-1)(n-2)}{N} P_{n-1}-\frac{\alpha n(n-1)}{N} P_{n}+(n+1) P_{n+1}-n P_{n} .
$$

Equation (3) should read as

$$
\partial_{t} G=\frac{k_{0}}{\langle k\rangle}[g(p)-1] G+(1-p) \partial_{p} G+\frac{\alpha}{N} p^{2}(p-1) \partial_{p}^{2} G .
$$

The paper has been corrected as of 22 June 2016. The text is incorrect in the printed version of the journal. 